\title{
DECIMAL/RESIDUE CONVERSION BY TIME-INTEGRATING CORRELATION
}

\author{
Demetri PSALTIS, Frank CAIMI, David CASASENT and Anastasios GOUTZOULIS \\ Carnegie-Mellon University, Department of Electrical Engineering, Pittsburgh, PA 15213, USA
}

Received 12 May 1980

\begin{abstract}
A time integrating acousto-optic correlator for residue arithmetic processing is described with experimental confirmation included. This system offers superior input and output space bandwidth product to space integrating systems described previously.
\end{abstract}

Optical residue arithmetic processors have received considerable attention $[1-4]$. These systems are attractive because they enable arithmetic operations to be performed without carries (and hence in parallel at high rates) and at high accuracy (without the need for components with large dynamic range). Optical systems are attractive for these processors because of their large time or space-bandwidth product and parallel processing features. In this paper, we utilize a correlation formulation [2] of residue arithmetic operations. Rather than using spatial pulse-position coding [1] to represent decimal and residue numbers, we employ a variant we refer to as temporal pulse-position coding. We describe a time integrating correlator and demonstrate its use in decimal-to-residue conversion. This system differs considerably from prior space-integrating correlators for optical residue processing [2] and offers superior input and output space-bandwidth product performance plus allows realization with available acousto-optic transducers with high bandwidth.

In residue arithmetic, an integer $J$ is represented by the $N$-tuple set of remainders or residues $\left(R_{m 1}, R_{m 2}\right.$, $\left.\ldots, R_{m N}\right)$ with respect to the $N$ integer moduli $\left(M_{1}\right.$, $M_{2}, \ldots, M_{N}$ ). The maximum integer value that can be represented by the $N$ moduli is $M-1$ where $M$ $=\prod_{i=1}^{N} m_{i}$. It is useful background to review the correlation processing used to convert $J$ into $R_{m}$ when spatial pulse position coding is used [2]. Consider a multichannel frequency plane correlator with input $g\left(x_{0}\right)$ $=\delta\left(x_{0}-J \Delta x\right)$, i.e. a delta function whose spatial location encodes $J$. Its Fourier transform $G(u)$ is incident on the frequency plane where a square-wave grating with fundamental frequency $u_{\mathrm{g}}$ is placed. The separation between orders in the transform of the grating is chosen to satisfy $m \Delta x=u_{\mathrm{g}} \lambda f_{\mathrm{L}}$, where $f_{\mathrm{L}}$ is the focal length of the transform lens. In the output correlation plane, we obtain

$f\left(x_{2}\right)=\sum_{n} \delta\left[x_{2}-(J-n m) \Delta x\right]$.

Since $R_{m} \Delta x=(J-n m) \Delta x$, we can aperture the region $0 \leqslant x_{2} \leqslant(m-1) \Delta x$ in the output plane and the spatial location of the output correlation peak in this region will be the desired $R_{m}$. When multiple gratings are present in the transform plane, multiple $R_{m i}$ outputs are obtained. In the general correlation description of this processor [2], $f=g \circledast h$, where $h\left(x_{0}\right)$ $=\Sigma_{n} \delta\left(x_{0}-n m_{i} \Delta x\right)$ is the system's impulse response.

Since an input plane resolution $M$ is necessary and since $M$ can become very large, we propose to use a time-integrating acousto-optic correlator and hence temporal pulse position coding to reduce input time bandwidth requirements. The proposed system is shown in fig. 1. It is a modified version of the wellknown time-integrating acousto-optic correlator [5] . Consider only the $x$ dimension of the system first. We describe the temporal light source modulation by $s_{0}(t)$ $=\delta(t-J \Delta t)$, where the time of occurrence of the pulse describes the input decimal number $J$ in temporal pulse position coding. The acousto-optic cell at $\mathrm{P}_{1}$ is uniformly illuminated with light modulated as $s_{0}(t)$. The acousto-optic cell with transit time $m \Delta t$ is fed with a 


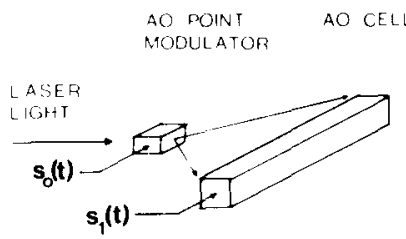

P P

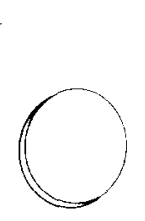

$L_{1}$

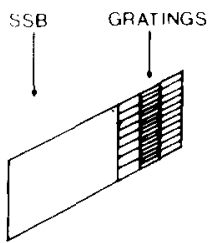

P

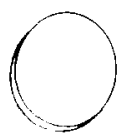

$L_{2}$

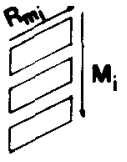

P

Fig. 1. Time integrating correlator for decimal-to-residue conversion using time pulse-position coding.

signal that consists of pulses of width $\Delta t$ and period $m \Delta t$, where $m$ is the residue modulus. The transmittance of $\mathrm{P}_{1}$ is $s_{1}(x, t)=\Sigma_{n} \delta(x-n m \Delta t+t)$. The $s_{0} s_{1}$ pattern leaving $P_{1}$ is imaged onto $P_{3}$ and time integrated on the $\mathrm{P}_{3}$ detector for $m \Delta t$. The horizontal output pattern at $\mathrm{P}_{3}$ is then the correlation $s_{0} \odot s_{1}$ and is again given by (1) with $\Delta x$ replaced by $\Delta t$. The spatial location (horizontally) of the output peak at $\mathrm{P}_{3}$ thus denotes the desired $R_{m}$ of $J$ modulo $m$.

To enable parallel conversion of $J$ into $R_{m i}$ for $N$ different bases $m_{i}$, we modify $s_{1}(t)$ and consider the $\mathrm{P}_{2}$ filter shown in fig. 1 and the vertical dimension of the $\mathrm{P}_{3}$ output plane. For $s_{1}(t)$ we use a sum of $N$ signals each consisting of pulses of width $\Delta t$ but with different periods $T_{i}=m_{i} \Delta t$ and different carrier frequencies $f_{i}$ within each $\Delta t$ for each moduli $m_{i}$. Each $f_{i}$ and hence each modulus signal appears spatially separated horizontally in the Fourier transform plane $\mathrm{P}_{2}$. At $\mathrm{P}_{2}$, we block the dc and minus first orders and pass only the plus first order by a single sideband aperture. Within this $\mathbf{P}_{2}$ aperture, we place $N$ vertical gratings. These cause the correlations $s_{0} \circledast s_{1 i}$ of $s_{0}$ with the different moduli $M_{i}$ signals at different $f_{i}$ to be spatially separated vertically at $P_{3}$. Thus the total $P_{3}$ pattern after

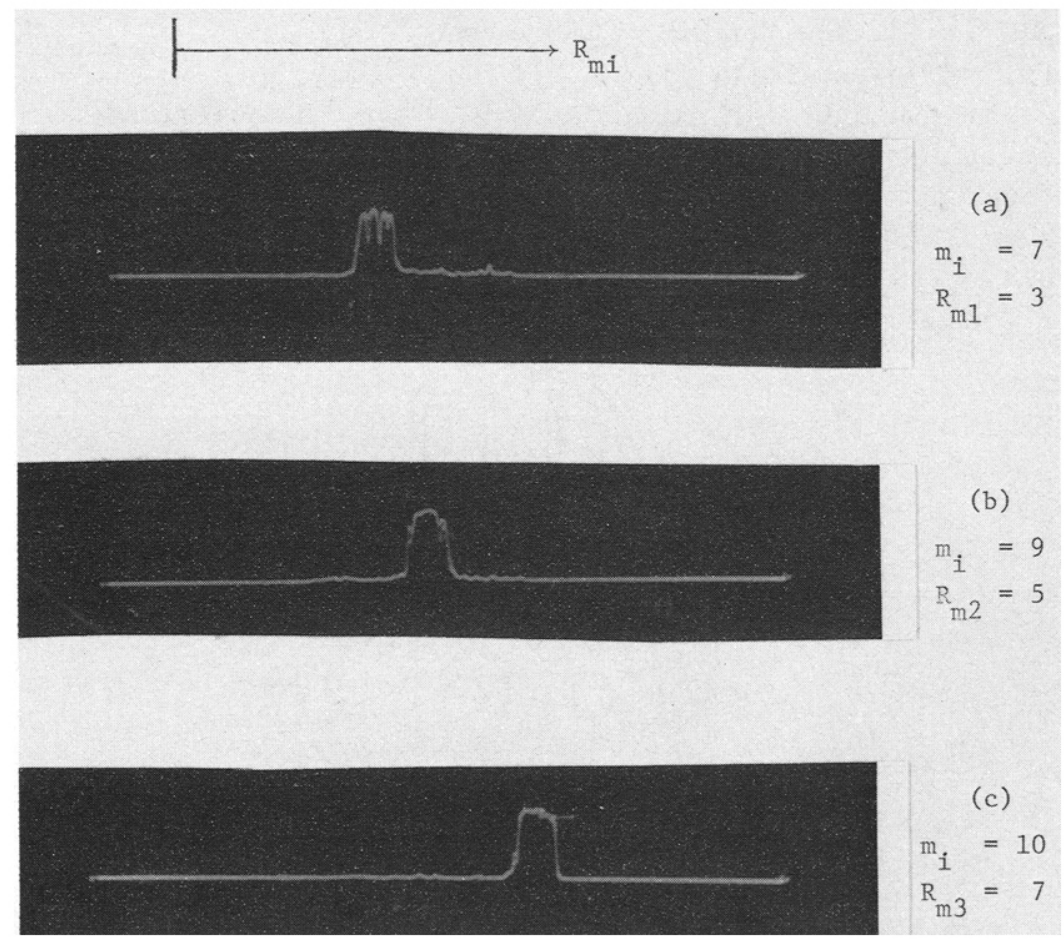

Fig. 2. Output $\mathrm{P}_{3}$ pattern in fig. 1 for a decimal input $J=437$ and moduli $m_{i}=7,9$ and 10 . 
time integration contains $N$ correlations $s_{0} * s_{1 i}$ on $N$ different horizontal lines. The horizontal position of each correlation peak corresponds to an $R_{m i}$ and the $m_{i}$ are encoded in the vertical output channels. Thus parallel multiple-moduli decimal-to-residue conversion is possible on the system of fig. 1 .

The system of fig. 1 was assembled. The acoustooptic cell used had a center frequency of $38 \mathrm{MHz}$, a bandwidth of $\pm 5 \mathrm{MHz}$ and a transit time of $32.25 \mu \mathrm{s}$. For the experimental demonstration, we chose three moduli: $m_{1}=7, m_{2}=9$ and $m_{3}=10$ giving a maximum $M \approx 630$. For the $s_{1}$ signal we chose $\Delta t=2 / 3.3$ $\mathrm{MHz}=606 \mathrm{~ns}$ and periods $T_{i}=m_{i} \Delta t=4.242,5.454$ and $6.060 \mu$ s with frequencies in individual pulses of $f_{i}=34.7,38$ and $41.3 \mathrm{MHz}$. For the sound velocity $v$ $=6.2 \times 10^{5} \mathrm{~mm} / \mathrm{s}$ of our acousto-optic cell, these three $f_{i}$ corresponded to spatial frequencies $f_{\mathrm{g} i}=f_{i} / v$ $=55.9,61.29$ and $66.61 \mathrm{cy} / \mathrm{mm}$. For the $\lambda=633 \mathrm{~nm}$ and $f_{\mathrm{L}}=762 \mathrm{~mm}$ system parameters used, a reasonable horizontal spatial separation of $2.62 \mathrm{~mm}$ was used between the gratings at $P_{2}$. The spatial frequencies chosen for the gratings at $P_{2}$ were $u_{\mathrm{g}}=15,35$ and 55 $\mathrm{cy} / \mathrm{mm}$ and the corresponding separation between the three linear detector arrays at $P_{3}$ was $9.65 \mathrm{~mm}$. In fig. 2 , we show the outputs from the three detector arrays at $\mathrm{P}_{3}$ for the case of a $J=437$ input. The locations of the output correlations are seen to lie at $R_{m i} \Delta x$ $=\left(J-n m_{i}\right) \Delta x=3 \Delta x, 5 \Delta x$, and $7 \Delta x$. A direct analysis confirms that the residues of $R_{m i}$ of $J=437$ modulo $m_{i}=7,9$ and 10 are 3,5 and 7 , thus confirming that the $\mathrm{P}_{3}$ output is as expected.

The $s_{1}$ signal and $\mathrm{P}_{2}$ mask can be fixed in this sys- tem as can the integration times $m_{i} \Delta t$ of the different linear detector arrays. In addition, only $m_{i}$ detectors are needed in each row and only $N$ columns of detectors are required to represent quite large numbers. When acousto-optic cells of larger bandwidths ( $1-5$ $\mathrm{GHz}$ is possible [6] ) are used, the required computation time becomes very fast. Extension of this time integrating correlator concept to residue-to-decimal converters, adders and multipliers is straightforward and follows the correlation formulation of residue arithmetic presented earlier [2].

The support of the National Science Foundation and motivation by RADC for this work is gratefully acknowledged. We thank M. Gottlieb of Westinghouse for fabricating the acousto-optic cell used.

\section{References}

[1] A. Huang, Y. Tsunoda and J.W. Goodman, Appl. Opties 18 (1979) 148.

[2] D. Psaltis and D. Casasent, Appl. Optics 18 (1979) 163.

[3] A. Tai, J. Cindrich, J. Fienup and C. Aleksoff, Appl. Optics 18 (1979) 2812.

[4] Proc. Soc. Photo-Opt. Instr. Engrs., Vol. 185, Optical Processing Systems (May 1979) and Vol. 232, 1980 Intern. Optical Computing Conf. (April 1980) (SPIE: Bellingham, Washington).

[5] R. Sprague and C. Koliopoulos, Appl. Optics 15 (1976) 89.

[6] Proc. Soc. Photo-Opt. Instr. Engrs., Vol. 180, Real-time Signal Processing-fI (April 1979) (SPIE: Bellingham, Washington). 